\title{
Identification of genomic loci associated with genotypic and phenotypic variation among Pseudomonas aeruginosa clinical isolates from pneumonia
}

\author{
Cristina S. Mesquita ${ }^{a}$, Pedro Soares-Castro ${ }^{a}$, Alberta Faustino ${ }^{b}$, Hugo M. Santos ${ }^{c, d}$, \\ José L. Capelo ${ }^{\mathrm{c}, \mathrm{d}}$, Pedro Santos ${ }^{\mathrm{a}, *}$ \\ ${ }^{a}$ CBMA - Centre of Molecular and Environmental Biology, Department of Biology, University of Minho, Campus de Gualtar, 4710-057, Braga, Portugal

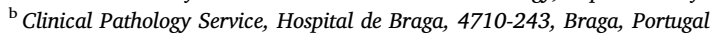 \\ ${ }^{c}$ UCIBIO, REQUIMTE, Departamento de Química, Faculdade de Ciências e Tecnologia, Universidade Nova de Lisboa, 2829-516, Caparica, Portugal \\ d PROTEOMASS Scientific Society, Madan Park, Rua dos Inventores, 2825-152, Caparica, Portugal
}

\section{A R T I C L E I N F O}

\section{Keywords:}

Pseudomonas aeruginosa

epidemiological study

Genotype profiling

Virulence factors

Antimicrobial resistance

Comparative genomics

\begin{abstract}
A B S T R A C T
In this work, a genotype-phenotype survey of a highly diversified Pseudomonas aeruginosa collection was conducted, aiming to detail pathogen-associated scenarios that clinicians face nowadays. Genetic relation based on RAPD-PCR of 705 isolates, retrieved from 424 patients and several clinical contexts, reported an almost isolatespecific molecular-pattern. Pneumonia-associated isolates HB13 and HB15, clustered in the same RAPD-PCR group, were selected to evaluate the genomic background underlying their contrasting antibiotic resistance and virulence. The HB13 genome harbors antibiotic-inactivating enzymes-coding genes (e.g. $a a c(3)-I a, a r r, b a_{V I M-2}$ ) and single-nucleotide variations (SNVs) in antibiotic targets, likely accounting for its pan-resistance, whereas HB15 susceptibility correlated to predicted dysfunctional alleles. Isolate HB13 showed the unprecedented rhlcluster absence and variations in other pathogen competitiveness contributors. Conversely, HB15 genome comprises exoenzyme-coding genes and SNVs linked to increased virulence. Secretome analysis identified signatures features with unknown function as potential novel pathogenic (e.g. a MATE-protein in HB13, a protease in HB15) and antibiotic resistance (a HlyD-like secretion protein in HB13) determinants. Detection of active prophages, proteases (including protease IV and alkaline metalloproteinase), a porin and a peptidase in HB15 highlights the secreted arsenal likely essential for its virulent behavior. The presented phenotype-genome association will contribute to the current knowledge on Pseudomonas aeruginosa pathogenomics.
\end{abstract}

\section{Introduction}

Pseudomonas aeruginosa is a versatile Gram-negative bacterium widespread throughout the environment, known as a ubiquitous opportunistic pathogen able to cause both acute and chronic infections in humans [1]. According to point-prevalence surveys carried out in USA, Europe and Asia, $P$. aeruginosa has emerged as a leading cause of nosocomial infections, being one of the primary agents to cause infections in immunocompromised individuals and other highly vulnerable patients [2-4]. Infections are often associated with great morbidity and mortality due to its wide spectrum resistance to antimicrobials. Therapeutic options are becoming increasingly limited with the continued emergence and spread of multidrug-resistant (MDR) strains, making it one of the most problematic organisms to treat and eradicate from hospital environment [5].

The advent of next-generation sequencing technologies prompted the expansion of genome-based knowledge of $P$. aeruginosa strains as several whole-genome projects have been completed and more are in completion [6]. Pseudomonas aeruginosa genome is a mosaic of a conserved core component, interrupted by combinations of strain-specific blocks of genetic material, acquired by horizontal gene transfer (HGT) events [7], that varies according to the evolutionary traits of each isolate. HGT events play an important role in the evolution of pathogen competitiveness and fitness, leading to a constant and unpredicted fluctuation of the clinical outcome [8]. Genome-wide comparisons between strains enabled the large-scale detection of genetic variability among isolates through identification of single-nucleotide variations (SNVs) and insertions/deletions of bases (indels), considerably improving the understanding of $P$. aeruginosa strain-specific physiology towards distinct pathogenesis traits.

A collaboration set with a Portuguese Hospital towards the elucidation of genotypic and phenotypic variability among $P$. aeruginosa

\footnotetext{
* Corresponding author.

E-mail address: psantos@bio.uminho.pt (P. Santos).
} 
clinical isolates, aimed to rouse a holistic research approach to provide relevant information and tools for the clinicians to circumvent the multi-resistance and pathogenicity phenomena in $P$. aeruginosa isolates. Currently, our Pseudomonas aeruginosa collection contains 705 isolates, seasonally collected from diverse clinical contexts, to provide a comprehensive epidemiological and genotypic categorization, contributing to an increased effectiveness of surveillance systems, outbreak detection and clinical treatment. To accomplish this task, we have tested and validated a PCR-based genotypic pipeline, as it is a fast and cost-effective method used for the primary screening of large collections [9].

In this work, from a representative subpanel further assayed for phenotypic comparison, two pneumonia-associated isolates were selected for high-throughput sequencing to identify the genomic basis associated with the distinct phenotypes, using both MiSeq and Genome Analyzer IIx [10] from Illumina. This is, to our knowledge, the first epidemiological report of a diverse collection of Portuguese Pseudomonas aeruginosa clinical isolates coupling genotyping, phenotyping and genomic approaches.

\section{Materials and methods}

\subsection{Pseudomonas aeruginosa isolates}

A Portuguese Hospital setting (Hospital de Braga) provided 705 Pseudomonas aeruginosa isolates retrieved from different sources during March and June 2010-2014, upon approval by the Hospital Bioethics Commission. Clinical isolates were obtained from different tissues/ fluids of 424 anonymous patients during routine clinical diagnostics practices, at the hospital pathology department, in accordance with approved guidelines. Information regarding source, hospital department, clinical context, date of isolation, patient age and gender was registered for each isolate. For comparison purposes, $P$. aeruginosa reference strain PAO1 was used, as it is the most used $P$. aeruginosa laboratory strain, allowing comparison to other studies.

\subsection{Diversity analysis by a standardized PCR-based genotypic pipeline}

After optimization (Supplementary Material A), a standardized RAPD-PCR-based genotypic pipeline, resorting to open access bioinformatic tools, was implemented. To standardize PCR amplifications over time, in each gel at least two lanes with molecular weight standards were loaded for an improved molecular weight approximation, along with a negative control (without DNA), and an amplification of the same reference isolate, in order to verify the obtained pattern. Every isolate was genotyped at least three times to confirm pattern-profile reproducibility.

RAPD-PCR groups were determined applying a similarity threshold to the clustered collection, and their representativeness and epidemiological frequency distribution throughout the years assessed. Results were explored associating groups to specific epidemiological profiles, to determine variability within isolates.

\subsection{Genotype-phenotype correlation of a Pseudomonas aeruginosa subpanel}

A panel of six clinical isolates (HB3, HB4, HB5, HB6, HB13, and HB15) and the Pseudomonas aeruginosa reference strain PAO1 were chosen for phenotype characterization: antibiotic susceptibility, motility, biofilm formation and pyocyanin production (Supplementary Material A). Extracellular protein content was assayed for the two isolates selected for genome sequencing.

\subsection{Next-generation genome sequencing}

Genomic DNA from a pan-resistant (resistant to all antibiotics tested) $P$. aeruginosa, isolate $\mathrm{HB13}$, and a susceptible $P$. aeruginosa, isolate HB15, was extracted and purified using the Wizard Genomic Purification Kit (Promega). Samples were processed according to Illumina instructions generating pair-ended libraries, sequenced using the Illumina MiSeq system (2x250 bp) at Yale Center for Genomic Analysis. Supplementary Material A details the followed pipeline for genome assembly, annotation and analysis. The Whole Genome Shotgun projects of Pseudomonas aeruginosa HB13 and HB15 have been deposited at EMBL/GenBank under the accessions AEVV00000000 and AEVW00000000, respectively. The versions here described are AEVV00000000.3 and AEVW00000000.3.

As antibiotic resistance and virulence greatly affect patient condition following infection, a detailed genomic contextualization was carried out focusing on $P$. aeruginosa genes related to resistance and virulence determinants, according to VFDB (Virulence Factors of Pathogenic Bacteria Database [11]), PHIDIAS (Victors Virulence Factors; http://www.phidias.us/victors), PseudoCAP [6] and CARD (Comprehensive Antibiotic Resistance Database [12]). This analysis included genome comparison, identification of distinctive genome traits and SNVs and/or indels in these determinants.

\subsection{Extracellular peptide mass fingerprinting}

Cell-free $P$. aeruginosa HB13 and HB15 supernatants were collected for protein extraction and identification: exponential phase $(4 \mathrm{~h})$, early stationary phase $(8 \mathrm{~h})$, late stationary phase $(12 \mathrm{~h})$ and dead zone $(20 \mathrm{~h})$. Supplementary Material A contains the detailed protocol, parameters and in silico tools used for peptide identification and analysis.

As we wanted to analyze the $P$. aeruginosa secretome (i.e. complete set of secreted proteins), identified proteins with predicted cytoplasmic localization were disregarded if no signal peptide cleavage site was identified. These cytoplasmic proteins are likely contaminants, nevertheless, natural $P$. aeruginosa autolysis could lead to these protein release or even secretion by non-traditional pathways.

\section{Results}

\subsection{Epidemiological and genetic diversity of the Pseudomonas aeruginosa collection}

This study provides an epidemiological overview of $P$. aeruginosa infections in a Portuguese Hospital setting coupled to genotypic categorization of 705 clinical isolates collected from a variety of infection loci, clinical context, hospital department and age groups from 424 patients (Fig. 1, Supplementary Table S1).

Regarding the infection context, about $49 \%(n=343)$ of isolates were collected from multi-infected loci, predominantly with Enterococcus faecalis, Escherichia coli, Staphylococcus aureus and Klebsiella pneumoniae (Fig. 1), posing additional clinical challenges in the eradication of infection focus. Isolates were categorized according to antimicrobial susceptibility as MDR (resistant to three or more antibiotic classes) and susceptible (no remarkable antibiotic resistance), classifying $28 \%$ as MDR isolates $(n=194$, Fig. 1 ), from which $11 \%$ $(\mathrm{n}=21)$ were classified as PDR (pan-resistant; generalized resistance). The frequency of the $P$. aeruginosa MDR phenotype in Portuguese Hospitals is higher than those reported in Europe or worldwide. According to the ATLAS (Antimicrobial Testing Leadership And Surveillance) database from Pfizer (https://atlas-surveillance.com/), in the same timeframe as our collection (2010-2014) and considering resistance to the assayed antibiotic classes, 13 and $14 \%$ of reported $P$. aeruginosa evidenced the MDR phenotype worldwide and in Europe, respectively, comparing to the $23 \%$ registered for Portugal. As mentioned by Castañeda-Montes et al. [13], this observed higher resistance could be owed to the socio-political context of the country, accounting for the national effort to improve antibiotic stewardship.

Epidemiological studies are fundamental to public health, mainly in 


\section{Patient age and gender}
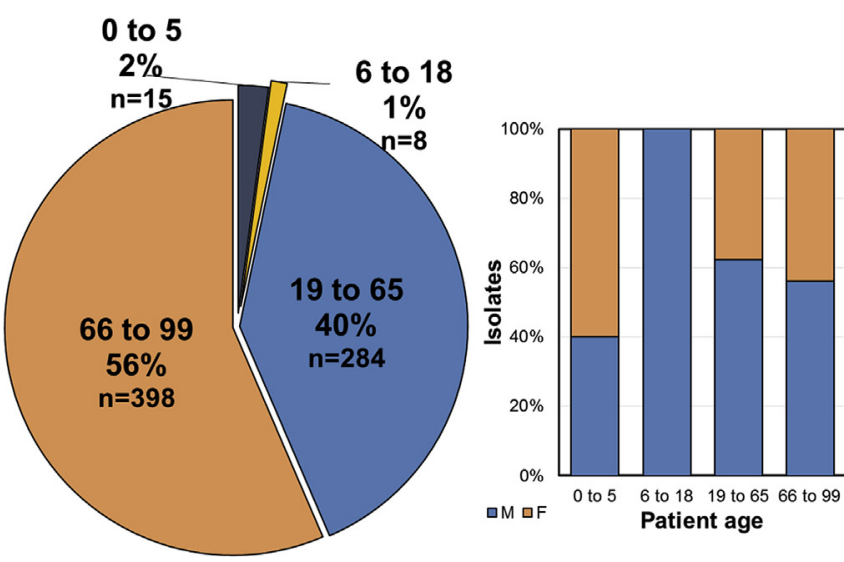

\section{Type of infection}

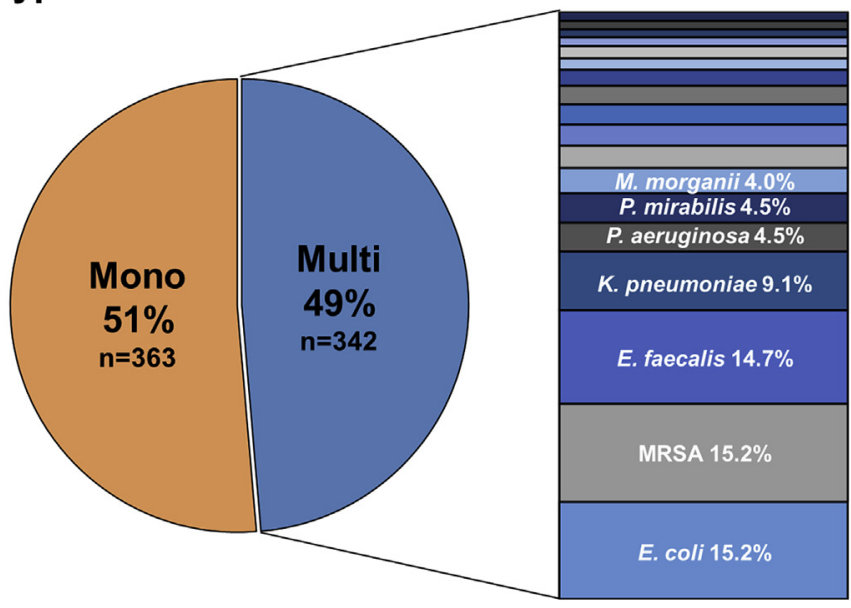

Drug resistance profile
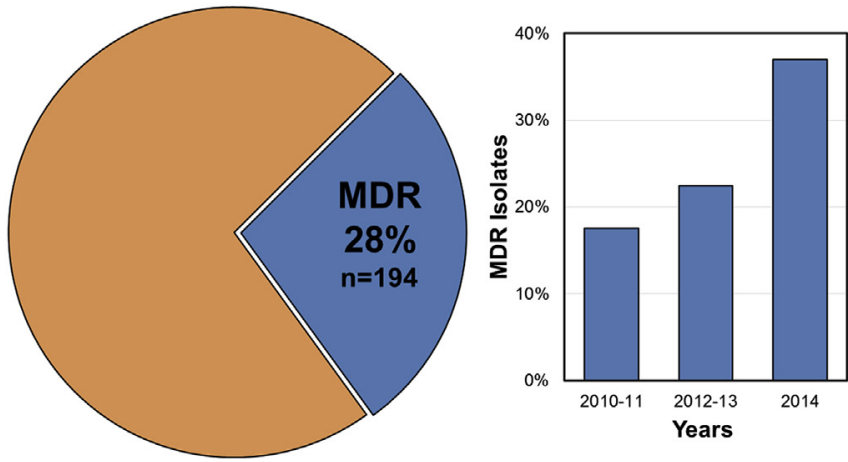

Fig. 1. Overall distribution of Pseudomonas aeruginosa isolates from 424 patients of a Portuguese Hospital setting, during March and June 2010-2014, according to age group and gender of the infected patient, type of infection and antibiotic resistance profile. Main co-inhabitant bacteria, when in a multi-infection context, as well as distribution of multidrugresistant isolates throughout the years are represented. $P$. aeruginosa isolates assayed for resistance to following antibiotics: ceftazidime, meropenem, piperacillin with tazobactam, tobramycin, amikacin, gentamicin and ciprofloxacin. Multidrug-resistance (MDR) phenotype attributed if resistant to three or more antibiotics classes.

microbiological control and preventive medicine, thus, we further categorized the observed heterogeneity of Pseudomonas aeruginosa clinical contexts. In this context, RAPD-PCR and combination of the Dice dissimilarity coefficient with the hierarchical clustering method UPGMA were tested and validated as the best strategy for a genotypic categorization of the $P$. aeruginosa collection (Supplementary Material $\mathrm{B}$ ), evidencing greater discriminatory power than other data analysis approaches, while clustering putative clonal isolates. RAPD-PCR was the technique chosen for genotypic characterization in our research since it is simple, inexpensive and rapid, less labor intensive and therefore more amenable to high-throughput screenings, allowing continuous monitoring of clinical isolates throughout the years.

Applying this PCR-based pipeline to the $P$. aeruginosa collection and considering a similarity threshold of $80 \%$, we observe a ratio of 0.94 genotypic profile per isolate (666 unique genotypic patterns for 705 samples) and a genetic relation ranging between 0.00 and 0.77 , sustaining an apparent great genetic variation. Most studies state a lesser distance, as the report of 35 genotypes for 385 isolates (ratio of 0.09 ) [14] and 31 genotypes for 48 isolates (ratio of 0.65) [15], however, they focus on specific isolates groups, such as ones recovered from CFpatients and not a diverse collection as ours.

In order to obtain a workable number of RAPD-PCR groups, a less conservative similarity threshold of $30 \%$, thus considering an apparent genetic variability of 0.70 , clusters the collection into 14 groups (Fig. 2) with distinct representativeness over the years and among multi-infection context and MDR phenotype, evidencing the great heterogeneity of $P$. aeruginosa population (Fig. 3, Supplementary Table S1). No obvious correlation of a RAPD-PCR group with a specific phenotype or source suggests a highly diversified non-nosocomial origin of infection for most clinical cases in this Portuguese Hospital setting, rather than epidemic or prevalent nosocomial cause, which sustains the well-described Pseudomonas aeruginosa ubiquitous character [1,16].

\subsection{Correlating genotype-phenotype in a Pseudomonas aeruginosa subpanel}

Understanding the reported $P$. aeruginosa individuality based on distinct genetic patterns is critical for correct infection diagnosis and treatment since clinicians rely heavily on predicting phenotypes from genetic variations [17]. In this context, a subpanel of clinical isolates and $P$. aeruginosa PAO1 as a reference strain were assayed for common virulence-associated traits, to verify a possible genotype-phenotype link. This subpanel comprised two same-source isolates genotypically clustered in the same RAPD-PCR group and one genotypically distant same-source isolate, from the two registered main sources: urine (HB3, HB5, HB6) and sputum (HB4, HB13, HB15) (Table 1). Thus, with this subpanel we assayed six isolates previously clustered in 4 groups, representing a ratio of 0.67 genotypic profiles per isolate, with an overall apparent genetic variability of 0.75 . Antibiotic resistance profiles ranged from pan-susceptibility (HB4, HB6, HB15), to a PDR phenotype (HB13).

Under the tested conditions, comparing to $P$. aeruginosa PAO1, the panel of isolates showed distinct motility profiles (Table 1). Isolate HB4 presented the largest flagella-mediated swimming halo and isolate HB15 showed the smallest. Ability to swarm was very asymmetric and, as all isolates displayed flagella-mediated swimming, differences in swarming may be associated with diversity in type IV pili biogenesis [18]. Concomitantly, isolates HB5 and HB6 exhibited the highest twitching motility halos and the highest swarming ability, whereas the other isolates showed reduced or lacked this kind of motility. Motility influences biofilm formation at different stages and at initial stages, motility is indeed crucial for biofilm architecture [19]. When comparing to $P$. aeruginosa PAO1, isolates HB5 and HB6 displayed higher biofilm production, which correlates to longer diameters detected in type-IV pili-dependent swarming and twitching assays. Pyocyanin is a blue pigment secreted by $P$. aeruginosa, described to act as virulence factor in infection [20]. Isolates HB3, HB4, HB15 and PAO1 (with no significant differences) had highest pyocyanin levels whereas isolate HB13 registered an undetectable amount of blue pigment, even after longer growth periods. We normalized and compiled these phenotypic traits in a multivariate matrix to compare with the genotype-based clustering (Fig. 4). The differential isolate grouping is easily observed 


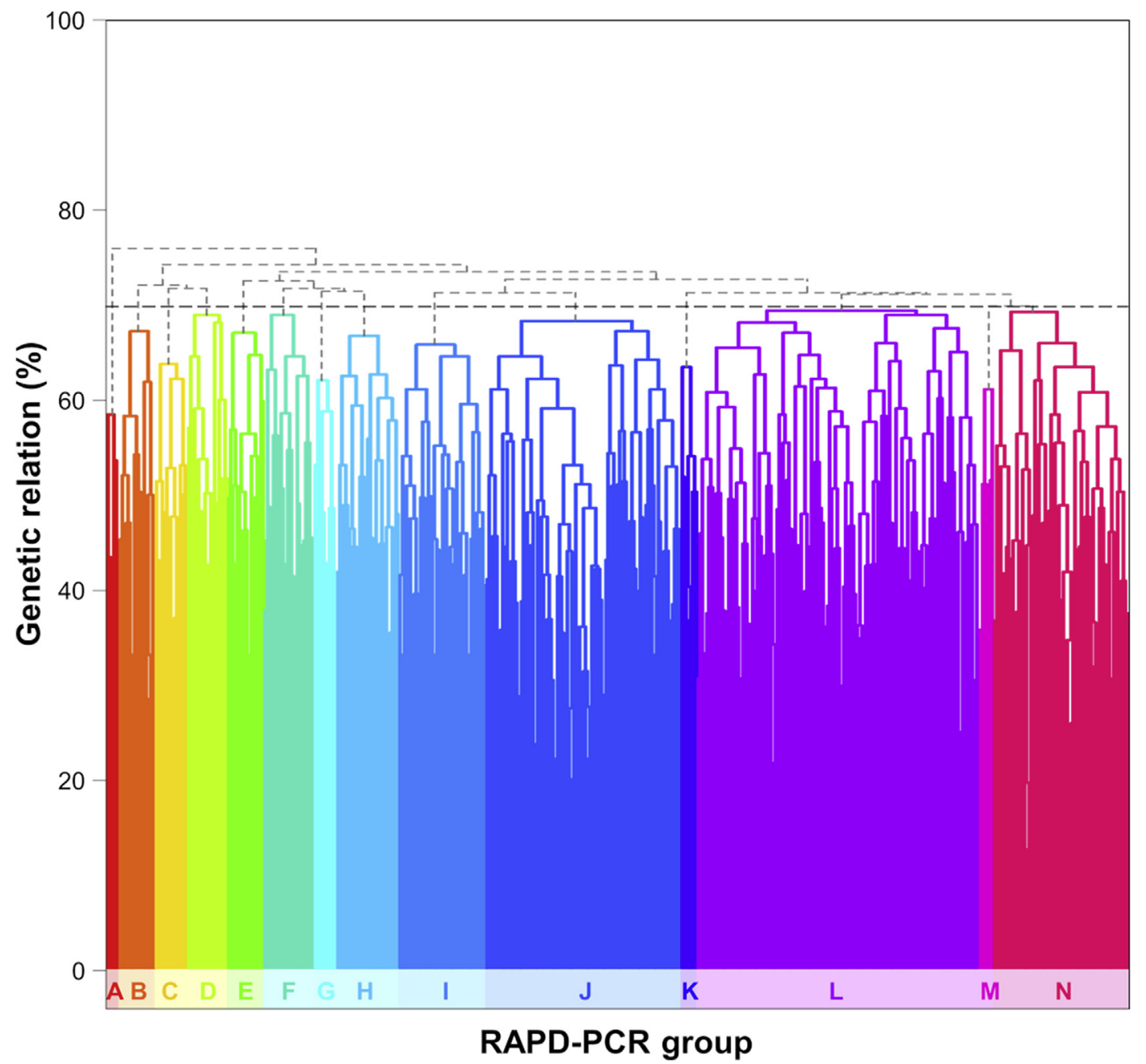

Fig. 2. Distribution of the Pseudomonas aeruginosa clinical isolates and reference strains by the 14 RAPD-PCR groups. Color represents distinct groups considering a genetic relation $h$ value of 0.70 (similarity of $30 \%$ ).

and the calculated correlation value of -0.328 suggests a lack of collinearity between the two trees, thus supporting $P$. aeruginosa heterogeneity.

The number of $P$. aeruginosa clinical isolates associated with respiratory infections corresponds to $29 \%$ of all Portuguese isolates, being pneumonia the main representative. In this study, isolates HB13 and HB15, clustered in the same RAPD-PCR group, out-performed others respectively concerning pan-resistance and virulence indicators. Having in mind the distinctive traits evidenced by isolates causing similar infections and the significant clinical impact, as the genotype discrimination was insufficient, isolates HB13 and HB15, both collected from the sputum of pneumonia-diagnosed patients and belonging to group L, were selected to determine their genomic background and its relationship with the registered phenotypic differences.

\subsection{Pseudomonas aeruginosa HB13 and HB15 comparative genomics}

P. aeruginosa HB13 and HB15 were sequenced with Illumina MiSeq technology and Supplementary Material A outlines the assembly pipeline. Scaffolding and gap-closure were performed using the MiSeq datasets here obtained (2x250 bp) and Illumina GAIIx 2x50 bp datasets previously reported [10]. The draft genome assembly was obtained after automated assembly improvement and manual curation. The isolate HB13 draft genome comprised 22 scaffolds (24 contigs) with 6,534,891 bp and 332 unknown bases (Ns), whereas the isolate HB15 draft genome comprised 19 scaffolds (20 contigs) with 6,643,276 bp and 654 Ns. This resulted in great contiguity and overall assembly quality improvement over the previously reported assembly.

Despite not being from a respiratory infection context, given that our data showed lack of genotypic pattern and source relation, $P$. aeruginosa PAO1 was selected as reference strain for comparative analysis (Fig. 5a) [21]. Freschi et al. [22] reported the division of 389 P. aeruginosa strains into three major groups, based on the SNPs within the core genome. Following this phylogenetic analysis, isolate HB13 was included in the same group as the $P$. aeruginosa PAO1, yet genetically closer to the reference strain LESB58, while isolate HB15 was included in the same group as $P$. aeruginosa PA14 (Fig. 5b). This corroborates the association obtained in this study of these two isolates with the reference strains LESB58 and PA14 (Supplementary Material B). However, since the same study reported that the conserved regions among all isolates represented $17.5 \%$ of the average $P$. aeruginosa genome size [22], our analysis was primarily focused on genomic features with distinct distribution.

The comparison of gene organization of isolates HB13 and HB15 with $P$. aeruginosa PAO1 reference genome allowed the identification of distinct distribution of 90 features related to antibiotic resistance and virulence in both isolates (10 and 80 genes, respectively; Fig. 6, Supplementary Table S2). Genetic loci associated with replacement islands (flagellin - RGP9; lipopolysaccharide O-antigen - RGP31; pilin RGP60; pyoverdine biosynthesis and posttranslational modification RGP73) were disregarded as they are under diversifying selection and are highly variable between strains [23]. In P. aeruginosa HB13, 72\% of 
RAPD-PCR groups representativeness

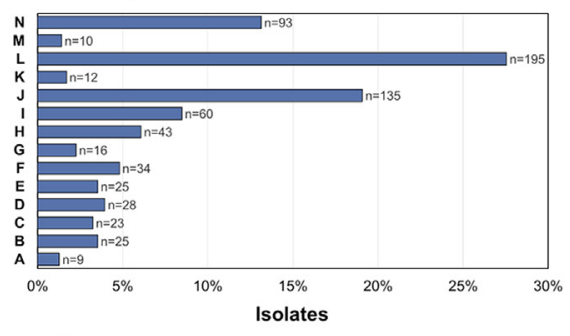

Isolation source ${ }^{a}$

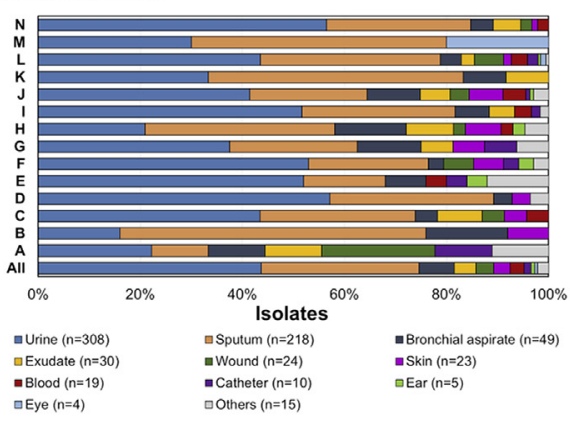

Multi-infections among RAPD-PCR groups

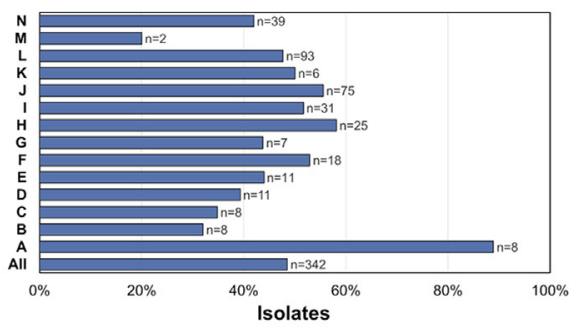

Clinical context ${ }^{b}$

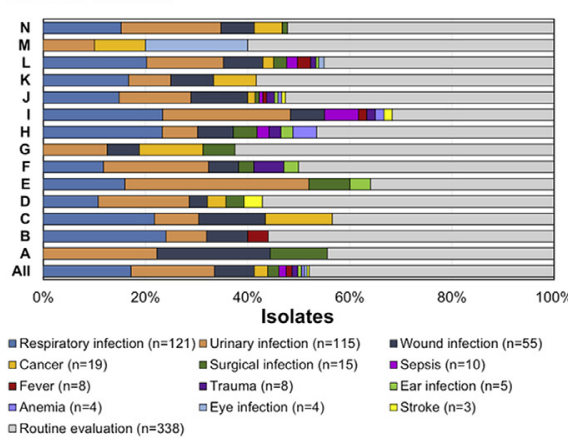

MDR isolates among RAPD-PCR groups

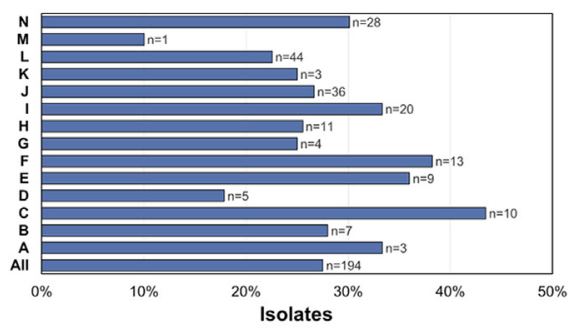

Hospital department a

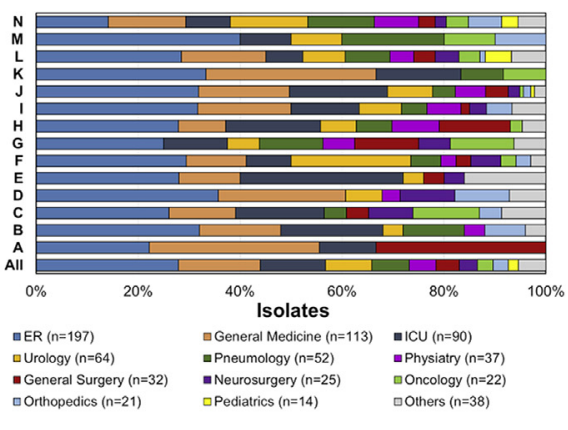

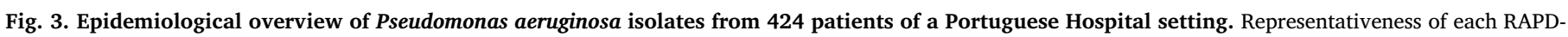

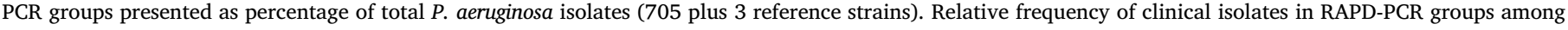

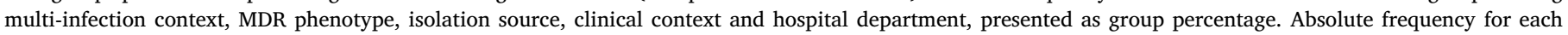
metadata, detailed in Supplementary Table S1, is indicated.

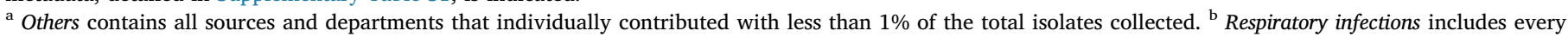

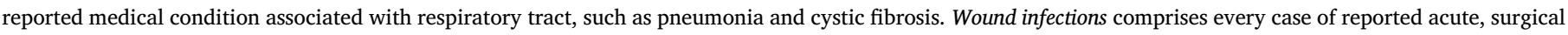
and chronic wounds, including ulcers. Routine evaluation corresponds to isolations in regular hospital visits, or not associated with a medical condition.

virulence determinants and $20 \%$ of antibiotic resistance features are missing, whereas isolate HB15 respectively lacks $16 \%$ and $80 \%$ of these determinants.

$P$. aeruginosa HB13 is missing several virulence-associated genetic features, such as those encoding for the transcriptional regulators ptxRS, that enhances the production of exotoxin A [24], and the rhlRI cluster, a key component of one $P$. aeruginosa Quorum-Sensing (QS) system. QS systems regulate, in a cell-density-dependent manner, several $P$. aeruginosa physiological traits, mainly related to virulence [25].
P. aeruginosa HB13 genome also lacks the AraC-type transcriptional regulator $v q s M$, a global regulator essential for $P$. aeruginosa QS and virulence modulation. Inactivation is related to lack of proteolytic activity owing to attenuated AHL production [26], pathogenesis loss and higher antibiotic resistance [27]. Moreover, the $a m b$ five-gene cluster responsible for the biosynthesis of L-2-amino-4-methoxy-trans-3-butenoic acid, a potent toxin against prokaryotes and eukaryotes with reported contribution to $P$. aeruginosa pathogenic potential [28], is also absent in isolate HB13. $P$. aeruginosa also secretes several exoenzymes

Table 1

Phenotypic characterization of a Pseudomonas aeruginosa subpanel from a Portuguese Hospital setting. This subpanel was assayed for antibiotic susceptibility, metallo- $\beta$-lactamases, motility, biofilm formation and pyocyanin production (Supplementary Material A). RAPD-PCR group is the classification according to genotypic clustering. Data from motility, biofilm and pyocyanin assays is shown as fold change ratio between values measured for each isolate and those of $P$. aeruginosa reference strain PAO1.

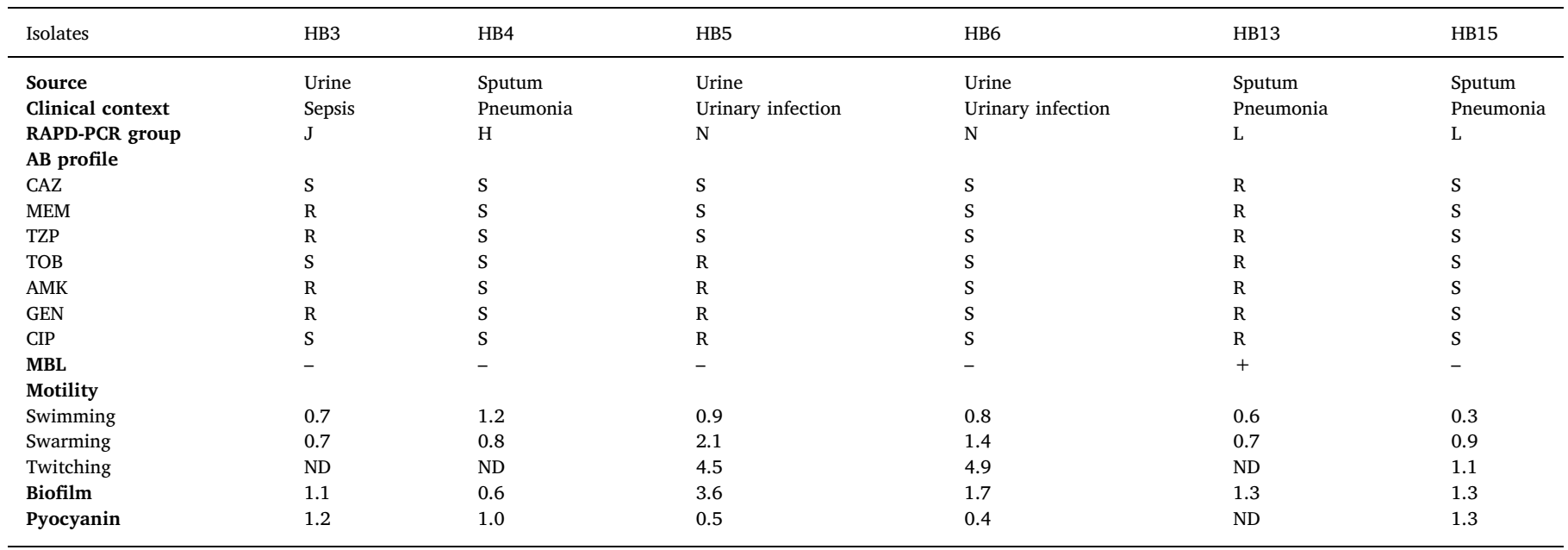

AB, Antibiotic; CAZ, Ceftazidime; MEM, Meropenem; TZP, Piperacillin/Tazobactam; TOB, Tobramycin; AMK, Amikacin; GEN, Gentamicin; CIP, Ciprofloxacin; S, susceptible; R, resistant.

MBL, metallo- $\beta$-lactamases; +, MBL detected; -, MBL not detected; ND, not detected. 

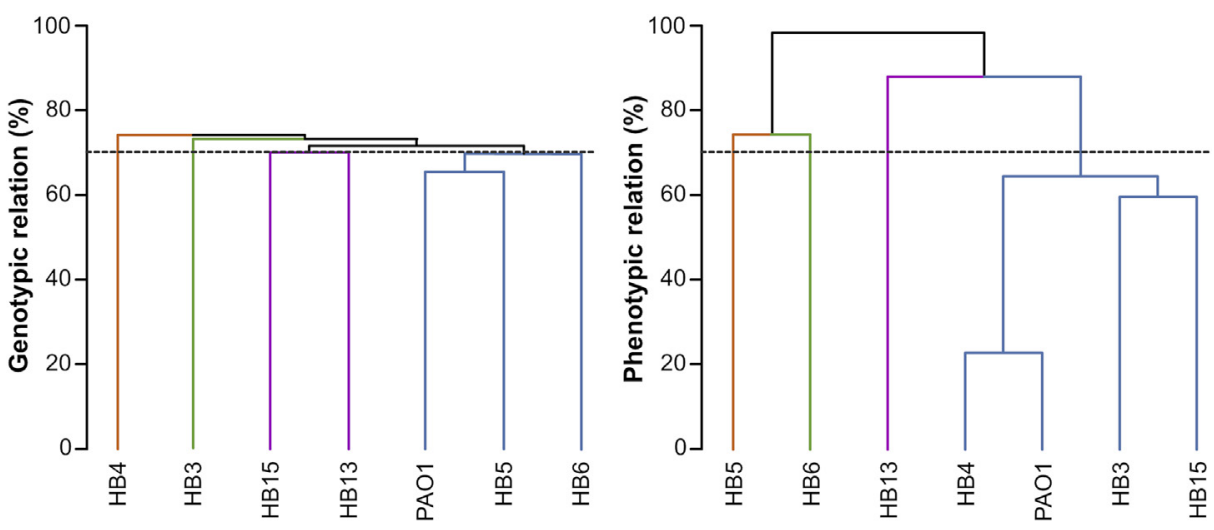

Fig. 4. Clustering of a Pseudomonas aeruginosa subpanel from a Portuguese Hospital setting. Dendrogram constructed based on genotypic and phenotypic relation, applying the previously described pipelines.

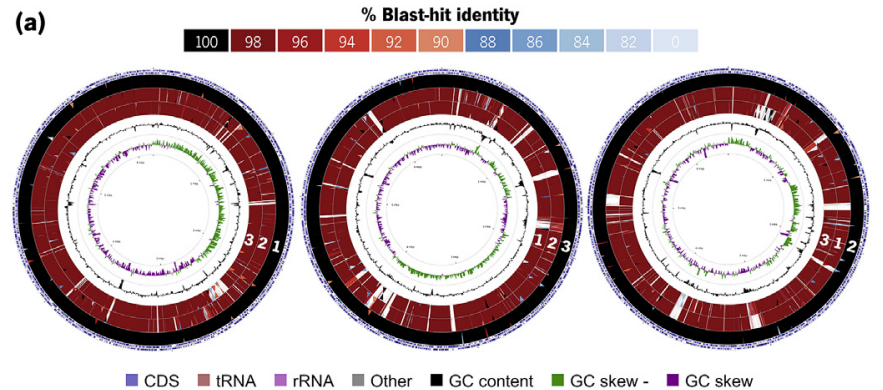

(b)

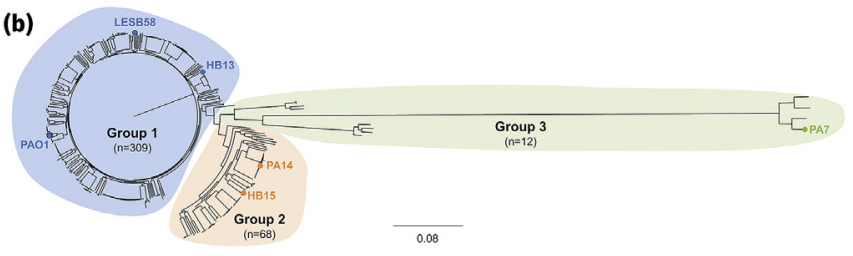

Fig. 5. Gene organization of Pseudomonas aeruginosa HB13 and HB15. (a) Graphical representation of BLAST-based genome comparison using CGView Comparison Tool [21]. Outermost circle depicts ORFs on the positive and negative strand. Innermost circles represent GC content and GC skew. (1) $P$. aeruginosa PAO1; (2) P. aeruginosa HB15; and (3) P. aeruginosa HB13. (b) Adaptation of the maximum likelihood tree of 389 Pseudomonas aeruginosa genomes based on SNPs within the core genome, reported by Freschi et al. [22]. Number of strains for each of the three major groups (group 1: blue, group 2: orange and group 3: green) is shown. Main $P$. aeruginosa reference strains and the two clinical isolates described in this study are highlighted.

that target host cells (ExoU, ExoS, ExoT, and ExoY), with differential effects in pathogenesis. ExoY is reported to have a minor role in pathogenesis [29], whereas ExoU is often associated with virulent $P$. aeruginosa strains and acute infections [30]. Furthermore, exoU deletion significantly reduces overall virulence and impairs pathogenesis development in the mice lung [31]. Studies on ExoS and ExoT report a lesser effect on virulence [32,33]. P. aeruginosa HB13 harbors only exoS and exoT genes, whereas $P$. aeruginosa HB15 harbors exoU, exoT and exoY genes.

We analyzed whether the genomic constitution of isolates HB13 and HB15 correlated with their antibiotic resistant phenotype by looking to antibiotic-resistant features, and we showed this to be the case, since the genome of isolate HB15 is, apparently, missing some loci, as the gene $a a c(3)-I a$, which codes for an aminoglycoside-modifying enzyme, and the aminoglycoside response regulator gene arr. In contrast, isolate HB13 evidenced an enrichment in efflux pumps and other antibiotic resistance-associated features, such as VIM-2, the most-reported worldwide extended spectrum metallo- $\beta$-lactamase [34], which, seemingly, confers pan-resistance to $\beta$-lactams and the sulfonamides resistance gene sul1 [35]. The multidrug resistance phenotype of isolate HB13 had been previously hinted by its allelic sequence type [10]. $P$. aeruginosa HB13 is a representative of ST175, a widely disseminated type associated with multidrug resistance, while $P$. aeruginosa HB15 belongs to the ST560, a rare allelic sequence type not specific to the human host, also associated with animal and environmental strains $[36,37]$. ST175 has been reported as an internationally distributed VIM2 MBL-producing epidemic lineage [38,39] and as one of the most successful high-risk clones, grouping the majority of MDR $P$. aeruginosa strains [40], sustaining our in silico analysis.

While data relevant to antibiotic resistance and virulence is rapidly expanding, a considerable part of microbial genomes is still associated with proteins with hypothetical or unknown function. As this comparative analysis relies on curated databases of known determinants and pathogenicity is both combinatorial and multifactorial [41], currently unknown factors cannot be ruled out but would not be identified by this approach. Therefore, aiming to provide new insights into $P$. aeruginosa pathogenicity, signature features of isolates HB13 and HB15, i.e. present only on the respective isolate genome, were analyzed (data not shown). We predicted protein domains for hits with unknown or putative function resorting to in silico tools to identify new potential determinants of the observed phenotypic variation. This analysis predicted domains linked to antibiotic resistance for two signature features in isolate HB13: a MATE domain in PA13_1001085, and a metallo- $\beta$ lactamase domain in PA13_1011590. The role of metallo- $\beta$-lactamases has been discussed and similarly, members of the multidrug and toxin extrusion (MATE) family are also associated with MDR phenotype, functioning as drug/ $\mathrm{Na}^{+}$antiporters [42]. Moreover, in isolate HB15 three hypothetical proteins putatively hold virulence-associated domains: a nucleotidyltransferase AbiEii toxin in PA15_0312500; a transcriptional regulator AbiEi antitoxin in PA15_0312505; and a peptidase M91 in PA15_0330735. Together, the first two constitute a type IV bacteriostatic toxin-antitoxin innate immunity bacterial abortive infection (Abi) system [43]. Abi systems provide bacterial population resistance to phage infection [44] and from our analysis, the only completely assembled Pseudomonas genome possessing this system is Pseudomonas stutzeri SLG510A3-8 [45]. Peptidase M91 is a zinc metallopeptidases family which members are effector proteins that aid in inactivation of inflammatory response [46].

\subsubsection{SNVs and indels in Pseudomonas aeruginosa HB13 and HB15 core genome}

The profile of nucleotide changes may unravel additional genetic signatures of strain-specific microevolution driven by selective forces intrinsic to the colonized niche, eventually leading to accumulation of adaptive mutations [47]. Aiming to contextualize variation associated with antibiotic resistance and virulence, we focused this analysis on these determinants, identifying for isolate HB13, sequence alterations 

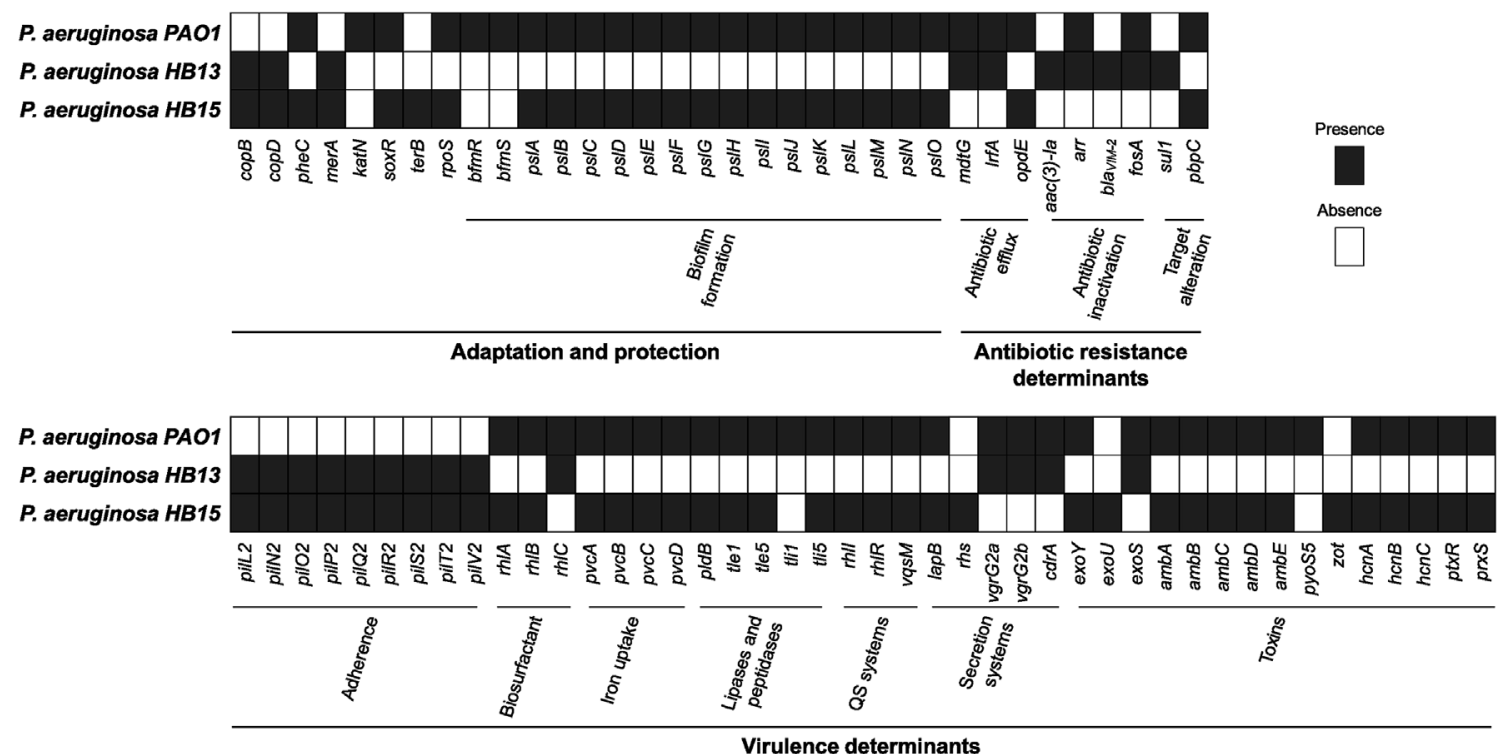

Virulence determinants

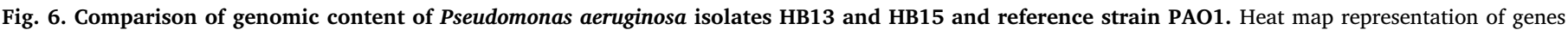

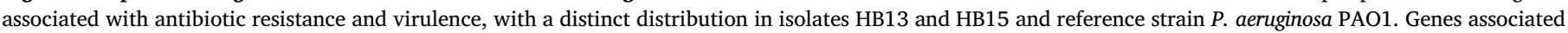

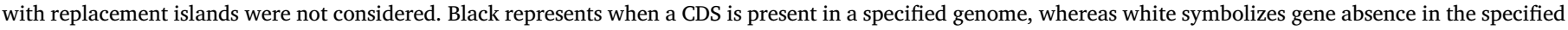
genome. Supplementary Table S2 identifies each corresponding locus_tag.

in 63 genes related to resistance and virulence altered, including 10 non-synonymous SNVs affecting protein function (Supplementary Table S3). A lesser virulent ability of $P$. aeruginosa HB13 was supported by intolerant SNVs in aprF and $a p r X$, suggesting a deficient secretion of alkaline protease [48], known to enhance ability of tissue invasion and interfere with host defense mechanisms [49] and in aaaA, where mutations significantly attenuate pathogenicity in the chronic wound model [50]. Regarding antibiotic resistance, predicted intolerant SNVs might also contribute to the isolate HB13 PDR-phenotype, such as the variation in the subunit B of DNA gyrase ( $g y r B)$, described in other fluoroquinolone-resistant $P$. aeruginosa isolates as a resistance determinant [51].

$P$. aeruginosa HB15 harbors in 46 genes linked to resistance and virulence, $6 \mathrm{SNVs}$ predicted to affect protein function and 7 indels (Supplementary Table S4). From the sequence alterations detected, the SNV in the aminopeptidase-coding gene $p h p A$ may contribute to an increased expression of virulence-associated alginate biosynthetic genes [52]. The observed susceptible phenotype of isolate HB15 might also be supported by variations in three specific gene products. The organic solvent tolerance protein OstA precursor is suggested to determine sensitivity to organic solvents, membrane permeability and susceptibility to antibiotics [53]. ArmZ, which interacts with MexZ, the negative-regulator of MexXY efflux [54], is unable to derepress MexXYrelated antibiotic resistance when dysfunctional, contributing to susceptibility to aminoglycosides. Furthermore, the membrane protein OpdE exhibited increased expression in resistant isolates [55] and mutation was associated with susceptibility to several antibiotics [56].

Further molecular approaches are necessary to evaluate the effect of these events in genes linked to improved resistance and virulence, towards circumventing $P$. aeruginosa multi-resistance and pathogenicity.

\subsection{Pseudomonas aeruginosa HB13 and HB15 pathogenic potential}

Despite all advantages of in silico tools, due to unavailability of experimental validations, there is a fair degree of uncertainty associated with its predictions. Therefore, in silico predicted avirulent PDR-phenotype of isolate HB13 and virulent antibiotic-susceptible phenotype of HB15 were analyzed by MALDI-TOF identification of extracellular protein content (Fig. 7, Table 2).
The main identified function-known proteins in PDR isolate HB13 supernatants corresponded to the secreted protease elastase LasB after $8 \mathrm{~h}$ of growth and in both exponential and stationary phases, the metallo- $\beta$-lactamase VIM-2, previously identified as a $P$. aeruginos $a$ HB13 signature feature (Fig. 6). Elastase LasB is a metalloprotease able to induce tissue damage [57] and involved in degradation of host tissuemodelling enzymes [58]. Metallo-ß-lactamases enzymes secretion causes $P$. aeruginosa antibiotic resistance [59], supporting in silico predictions and the evidenced isolate HB13 resistance to $\beta$-lactams.

Mass spectrometry of isolate HB15 supernatants detected several protein-degrading enzymes, such as an alkaline metalloproteinase precursor and a putative peptidase (PA15_0315610), within the exponential growth phase. $P$. aeruginosa HB15 evidenced proteolytic activity at early stages and an elastase LasB and protease IV enrichment over time. These extracellular protein/peptide-degrading enzymes produced by isolate HB15, namely elastase LasB, protease IV and alkaline metalloproteinase, are well-known $P$. aeruginosa virulence factors, secreted to assist bacterial invasiveness [60,61].

Blast analysis of an aminopeptidase, secreted by isolate HB13 (PA13_1011705) at $12 \mathrm{~h}$ of growth, and by isolate HB15 (PA15_0303145) at $8 \mathrm{~h}, 12 \mathrm{~h}$ and $20 \mathrm{~h}$ of growth, showed $100 \%$ identity to a leucine aminopeptidase PaAP, present in several other $P$. aeruginosa, with reported role in pathogenesis [62] and possibly contributing to infection persistence [63]. Despite both isolates being able to secrete it, $P$. aeruginosa HB15 did it from the early stationary phase to the dead zone, suggesting an earlier and more permanent virulent potential. Both $P$. aeruginosa $\mathrm{HB} 13$ and $\mathrm{H} 15$ also secreted a hypothetical protein (PA13_1021130, PA15_0314610) homologous to IMPa, an immunomodulating metalloprotease with suggested role in $P$. aeruginosa persistence and pathogenesis [64].

We identified a putative membrane protein (PA13_1009415) in isolate HB13 whose domain prediction associated with HlyD-like secretion proteins, particularly a component of a type I secretion system anchored in the cytoplasmic membrane [65], whose localization was confirmed by in silico analysis. This protein belongs to a large family of membrane fusion proteins involved in export of different compounds, including drug molecules [66], as does the MexA in P. aeruginosa [67], thus associating this hit with antibiotic resistance.

Isolate HB15 secreted a putative porin (PA15_0327485) predicted to 

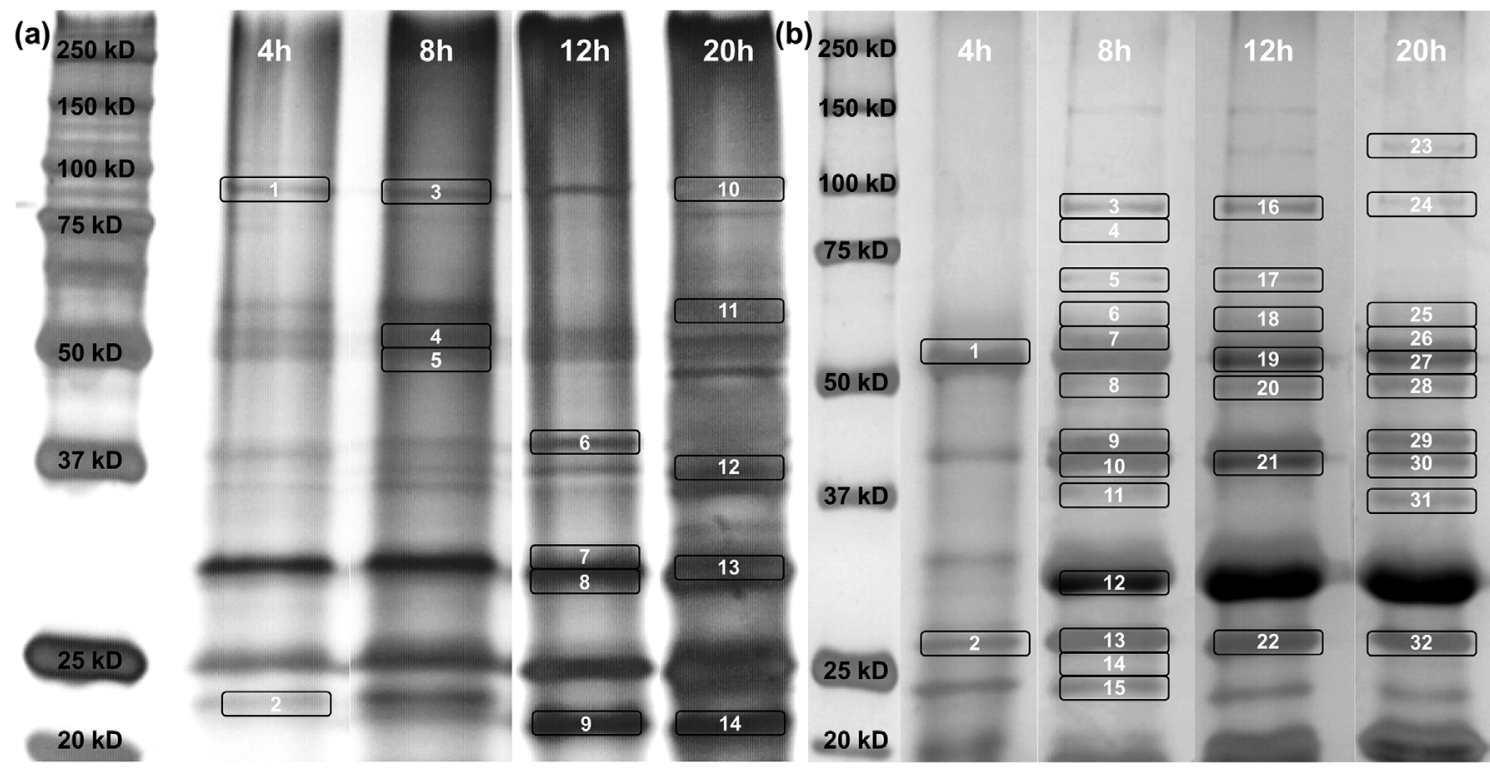

Fig. 7. Extracellular protein profiles of Pseudomonas aeruginosa isolates HB13 and HB15. Several time points were collected from cultures of $P$. aeruginosa isolates HB13 (a) and HB15 (b): exponential phase (4h), early stationary phase ( $8 \mathrm{~h})$, late stationary phase (12 h) and dead zone (20 h). Correspondence of gel coordinates and protein ID available at Table 2 and Supplementary Table S5.

be associated with OprF, a major outer membrane protein conserved across clinical isolates. Studies describe it as a host immune system sensor, modulating QS to enhance virulence when the bacteria are in contact with host cells [68], being required for expression of $P$. aeruginosa full virulence [69]. After $4 \mathrm{~h}$ of growth, isolate HB15 also secreted a putative peptidase (PA15_0315610), whose blast analysis indicated homology to proteins mainly belonging to other Pseudomonas species, some annotated as peptidase M16 (Supplementary Fig. S1). Accordingly, in silico tools predicted two peptidase M16 domains, one active and other inactive. Peptidase M16 is known to hold its substrate as a clamp, with an active peptidase domain and a C-terminal inactive domain [70], thus hinting a functional protein secretion by isolate HB15. These metalloproteases are important for several biological processes and its substrates are involved in human disease pathogenesis [71].

\section{Discussion}

Albeit the high number of diagnosed infections, our data suggests an almost unique molecular fingerprint for each clinical isolate. This genetic singularity may reflect $P$. aeruginosa success in human infections, with repercussions in patient segregation policies and therapeutic strategies. High heterogeneity of MDR (including PDR and colistin-resistant) isolates indicates that acquisition of these traits may not be more favorable to occur in genotypically close isolates, but rather a widespread ability. Inadequate procedures towards infection control and eradication policies were signaled by a radical change in resistance profile, towards MDR (e.g. susceptible isolate HB314 and MDR isolate HB334, collected from same-patient sputum within one month), which lead to bacterial survival and adaptation to therapeutic strategies. These are alarming findings given the inability to relate epidemiology

Table 2

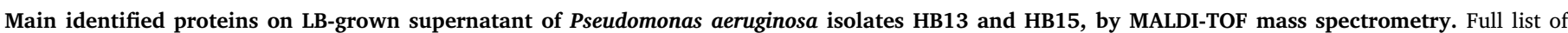
identified proteins and corresponding scores is available at Supplementary Table S5.

\begin{tabular}{|c|c|c|c|c|c|}
\hline Locus_Tag & Product & Predicted domains and superfamilies & $\mathrm{SP}^{\mathrm{b}}$ & Localization $^{\mathrm{c}}$ & $\mathrm{TP}^{\mathrm{d}}$ \\
\hline PA13_1004255 & Elastase LasB & - & + & $\mathrm{E}$ & $8 \mathrm{~h}, 12 \mathrm{~h}, 20 \mathrm{~h}(\mathrm{a} 4, \mathrm{a} 8, \mathrm{a} 13)$ \\
\hline PA13_1009415 & Putative membrane protein & $\begin{array}{l}\text { HlyD-like secretion proteins } \\
\text { RND eflux pump family, MFP }\end{array}$ & + & $\mathrm{CM}$ & $20 \mathrm{~h}(\mathrm{a} 11)$ \\
\hline PA13_1011705 & Aminopeptidase & - & + & $\mathrm{E}$ & $12 \mathrm{~h}(\mathrm{a} 7)$ \\
\hline PA13_1021130 & Hypothetical protein & Peptidase M60; DUF3712 & + & $\mathrm{OM}$ & $4 \mathrm{~h}, 8 \mathrm{~h}, 20 \mathrm{~h}(\mathrm{a} 1, \mathrm{a} 3, \mathrm{a} 10)$ \\
\hline PA13_1028635 & Metallo- $\beta$-lactamase VIM- 2 subclass B1 & - & + & $\mathrm{U}$ & $4 \mathrm{~h}, 12 \mathrm{~h}(\mathrm{a} 2, \mathrm{a} 9)$ \\
\hline PA15_0303145 & Aminopeptidase & - & + & $\mathrm{E}$ & 8 h, 12 h, 20 h (b7, b19, b27) \\
\hline PA15_0307120 & Elastase LasB & - & + & $\mathrm{E}$ & $8 \mathrm{~h}, 12 \mathrm{~h}, 20 \mathrm{~h}(\mathrm{~b} 12, \mathrm{~b} 18, \mathrm{~b} 25)$ \\
\hline PA15_0309435 & Protease IV & - & + & $\mathrm{E}$ & $8 \mathrm{~h}, 12 \mathrm{~h}, 20 \mathrm{~h}(\mathrm{~b} 13, \mathrm{~b} 22, \mathrm{~b} 32)$ \\
\hline PA15_0310815 & Alkaline metalloproteinase precursor AprA & - & + & $\mathrm{E}$ & $4 \mathrm{~h}(\mathrm{~b} 1)$ \\
\hline PA15_0314315 & Phage tail length tape measure protein & - & - & $\mathrm{OM}$ & $8 \mathrm{~h}(\mathrm{~b} 4)$ \\
\hline PA15_0314335 & Bacteriophage tail protein & Phage tail sheath protein subtilisin-like domain & - & $\mathrm{U}$ & 8 h, 12 h, 20 h (b9, b21, b29) \\
\hline PA15_0314340 & Bacteriophage protein & Phage tail-collar fibre protein & + & $\mathrm{E}$ & $8 \mathrm{~h}, 12 \mathrm{~h}(\mathrm{~b} 5, \mathrm{~b} 17)$ \\
\hline PA15_0314610 & Hypothetical protein & Peptidase M60; DUF3712 & + & $\mathrm{OM}$ & $8 \mathrm{~h}, 12 \mathrm{~h}, 20 \mathrm{~h}(\mathrm{~b} 3, \mathrm{~b} 16, \mathrm{~b} 24)$ \\
\hline PA15_0315610 & Putative peptidase & $\begin{array}{l}\text { Peptidase M16, N-terminal } \\
\text { Peptidase M16 inactive domain, C-terminal }\end{array}$ & + & $\mathrm{U}$ & $4 \mathrm{~h}(\mathrm{~b} 2)$ \\
\hline PA15_0327485 & Putative porin & OprF membrane domain & + & $\mathrm{OM}$ & $8 \mathrm{~h}(\mathrm{~b} 11)$ \\
\hline
\end{tabular}

a Prediction of protein domains according to InterPro, HMMER, CDD for hits with unknown or putative function.

b Prediction of signal peptide (SP) cleavage sites presence according to SignalP 4.1, TargetP 1.1 and TatP 1.0: , absence; + , presence.

c Prediction of cellular localization according to PSORTb, Gneg-mPLoc, SOSUIGramN, CELLO2GO: E, extracellular; OM, outer membrane; P, periplasmic; CM, cytoplasmic membrane; F, flagellar; U, unknown.

d TP, time point. The corresponding gel coordinate from Fig. 5 is indicated in parentheses. 
to genotypic profiles, sustaining the need for stricter infection control and effective antibiotic stewardship measures in hospital settings for better patient management. To further understand and evaluate the clinical consequences of such genetic diversity among $P$. aeruginosa, high-throughput approaches as those employed by the international Pseudomonas aeruginosa consortium [22] are mandatory. Expectedly, these efforts will contribute to the development of effective molecular biomarker-based tools for fast discrimination and diagnosis of individual $P$. aeruginosa isolates.

Ultimately, our work highlighted the genomic diversification occurring within $P$. aeruginosa strains and hinted that it might reflect a distinctive pathogenic behavior. Accordingly, apparent absence of virulence determinant genes and the exoenzyme-coding genes profile in $P$. aeruginosa HB13 suggests decreased ability to deploy extensive host damage, whereas $P$. aeruginosa HB15, putatively, possesses an arsenal that allows it to have a virulent behavior. Negative mutants in QS systems have reduced cytotoxicity due to defective signaling and reduced release of extracellular virulence factors [72]. However, these reported negative mutants display point mutations and not complete cluster deletions as deduced for the genomic analysis of isolate HB13. The rhlRI system directly activates the rhamnolipid biosynthesis operon rhlAB and hydrogen cyanide production by hcnABC [73], known contributors to in vivo $P$. aeruginosa competitiveness and also absent in isolate HB13. Moreover, mutations in rhRI systems are correlated to increased denitrification activity and therefore anaerobic growth in $P$. aeruginosa PAO1 [74]. Since reduced oxygen concentrations and anaerobiosis are frequently associated with lung environment and biofilm growth [75], rhlRI-deficiency would be beneficial for a chronic persistent strain. Isolate HB13 was able to establish lung infection in a pneumonia-diagnosed patient. Deletions of rhlRI-related operons might have occurred, driven by selective evolution during lung colonization. Absence of hcnABC [76] and rhlAB [77] clusters, deletions of rhlR or rhlI [78] have been described, particularly in persistent infections and with reported attenuated virulence. To our knowledge, this is the first report of a clinical isolate not carrying these three clusters.

SNVs and indels did not occur evenly spread through the genome of both isolates, suggesting that some genetic loci are highly conserved while others are hotspots, prone to variations that might translate into niche-specific adaptations or isolate-specific characteristics, thus partially constituting the molecular basis for the registered phenotypic variations.

Our in silico analysis supported the phenotypic characterization of both $P$. aeruginosa isolates, corroborating the PDR and avirulent phenotype of isolate HB13, and the pan-susceptible and higher virulent potential of isolate HB15. This strategy allowed the identification of $P$. aeruginosa putative novel determinants among the signatures features of both isolates, that would otherwise be ignored, making wholegenome sequencing a more unbiased and consistent method for determining the genetic potential for antibiotic resistance and virulence. Moreover, we found a set of, apparently, secreted proteins with previously unknown or putative function and our analysis suggests an involvement in $P$. aeruginosa pathogenesis. For instance, homology search of the peptidase secreted by isolate HB15 (Supplementary Fig. S1) showed that it is widespread in Pseudomonas aeruginosa and other human pathogens, as the Gram-negative Acinetobacter baumannii and Enterobacter cloacae, but also Gram-positive bacteria as Streptococcus pneumoniae, often found in co-inhabitation with Pseudomonas aeruginosa $[79,80]$, suggesting a HGT phenomenon. Homologous hits were also found in environmental bacteria, which does not discard a peptidase virulence-association, as some environmental strains are known plant pathogens with reported ability to infect humans [81]. Considering survival and adaptation of bacteria in humans similar to the one in the environment, pathogenicity determinants will also be present in non-pathogens. Future work should focus on characterization of the molecular activity of these secreted proteins with previously unknown or putative function as novel antibiotic resistance and virulence determinants.

Given the multifactorial and combinatorial nature of $P$. aeruginosa pathogenicity [41], secretion of more virulence factors by $P$. aeruginosa HB15 together with other observed phenotypic traits and cytotoxic data (data not shown), corroborate former in silico predictions of distinctive virulent behaviors for isolates HB13 and HB15. Active prophages, proteases and peptidases possibly play a determinant role in its modulation, particularly in the latter isolate.

\section{Conclusion}

Associating clinical outcome with phenotype and genotype is imperative to aid clinicians in treatment and eradication of bacterial infections, towards a more efficient and tailored approach to tackle isolate-specific singularity. Epidemiological, genotyping and phenotyping follow-up studies are required to verify the consequences of the clinical approach. Complementary diagnostic tests are crucial to guide clinicians to choose therapeutic strategies more appropriately, based not only on species identification and antibiotic resistance profiles but also in molecular patterns of detected pathogens. However, evaluating the genotypic profile might not provide the necessary information to account for a phenotype. In summary, as showed in this study, linking $P$. aeruginosa genomic repertoire to a phenotypic profile is a necessary aspect of uncovering novel pathogenic mechanisms and understanding the relative contribution of known determinants and their allelic variants.

Expanding this approach to other $P$. aeruginosa clinical isolates would aid in the creation of a variations profile and evolutionary strategies, correlating their genomic repertoire to a phenotypic profile. Combining available pipelines with the ever increasing -omics data will lead to a better prioritization strategy where the most promising determinants can be rapidly targeted for future analysis, aimed at better understanding $P$. aeruginosa pathogenesis. Integration of this data with the clinical outcome will deliver a solid description of the pathogen performance. This would rouse the establishment of a bacteriological, genome typing-like, tool that may help in diagnostic of Pseudomonas aeruginosa strains and in prognostic, giving the possibility to provide a customized antimicrobial treatment.

\section{Conflicts of interest}

The authors declare no conflicts of interest.

\section{Acknowledgments}

This work was supported by the strategic programme UID/BIA/ 0050/2013 (POCI-01-0145-FEDER-007569) funded by national funds through FCT I.P., by ERDF through the COMPETE2020 - Programa Operacional Competitividade e Internacionalização (POCI) and through a PhD grant (SFRH/BD/98558/2013) attributed to C.S.M. The facility for Biological Mass Spectrometry Isabel Moura was funded by Proteomass Scientific Society. H.M.S. is funded by the FCT 2015 Investigator Program (IF/00007/2015).

\section{Appendix A. Supplementary data}

Supplementary data to this article can be found online at https:// doi.org/10.1016/j.micpath.2019.103702.

\section{References}

[1] K.G. Kerr, A.M. Snelling, Pseudomonas aeruginosa: a formidable and ever-present adversary, J. Hosp. Infect. 73 (2009) 338-344.

[2] European Centre for Disease Prevention and Control (ECDC), Point Prevalence Survey of Healthcare Associated Infections and Antimicrobial Use in European Acute Care Hospitals, Stockholm, Sweden, (2013).

[3] S.S. Magill, J.R. Edwards, W. Bamberg, Z.G. Beldavs, G. Dumyati, M.A. Kainer, 
R. Lynfield, M. Maloney, L. McAllister-Hollod, J. Nadle, S.M. Ray, D.L. Thompson, L.E. Wilson, S.K. Fridkin, Emerging infections Program healthcare-associated infections and antimicrobial use prevalence survey team, multistate point-prevalence survey of health care-associated infections, N. Engl. J. Med. 370 (2014) 1198-1208.

[4] J.Y. Liu, Y.H. Wu, M. Cai, C.L. Zhou, Point-prevalence survey of healthcare-associated infections in Beijing, China: a survey and analysis in 2014, J. Hosp. Infect. 93 (2016) 271-279.

[5] U. Theuretzbacher, Accelerating resistance, inadequate antibacterial drug pipelines and international responses, Int. J. Antimicrob. Agents 39 (2012) 295-299.

[6] G.L. Winsor, E.J. Griffiths, R. Lo, B.K. Dhillon, J.A. Shay, F.S.L. Brinkman, Enhanced annotations and features for comparing thousands of Pseudomonas genomes in the Pseudomonas genome database, Nucleic Acids Res. 44 (2016) D646-D653.

[7] V. Kung, E.A. Ozer, A.R. Hauser, The accessory genome of Pseudomonas aeruginosa, Microbiol. Mol. Biol. Rev. 74 (2010) 621-641.

[8] M.W. Silby, C. Winstanley, S.A. Godfrey, S.B. Levy, R.W. Jackson, Pseudomonas genomes: diverse and adaptable, FEMS Microbiol. Rev. 35 (2011) 652-680.

[9] A. Doléans-Jordheim, B. Cournoyer, E. Bergeron, J. Croizé, H. Salord, J. André, M.A. Mazoyer, F.N.R. Renaud, J. Freney, Reliability of Pseudomonas aeruginosa semi-automated rep-PCR genotyping in various epidemiological situations, Eur. J. Clin. Microbiol. Infect. Dis. 28 (2009) 1105-1111.

[10] P. Soares-Castro, D. Marques, S. Demyanchuk, A. Faustino, P.M. Santos, Draft genome sequences of two Pseudomonas aeruginosa clinical isolates with different antibiotic susceptibilities, J. Bacteriol. 193 (2011) 5573.

[11] L. Chen, D. Zheng, B. Liu, J. Yang, Q. Jin, VFDB 2016: hierarchical and refined dataset for big data analysis - 10 years on, Nucleic Acids Res. 44 (2016) D694-D697.

[12] B. Jia, A.R. Raphenya, B. Alcock, N. Waglechner, P. Guo, K.K. Tsang, B.A. Lago, B.M. Dave, S. Pereira, A.N. Sharma, S. Doshi, M. Courtot, R. Lo, L.E. Williams, J.G. Frye, T. Elsayegh, D. Sardar, E.L. Westman, A.C. Pawlowski, T.A. Johnson, F.S.L. Brinkman, G.D. Wright, A.G. McArthur, Card 2017: expansion and modelcentric curation of the comprehensive antibiotic resistance database, Nucleic Acids Res. 45 (2017) D566-D573.

[13] F.J. Castañeda-Montes, M. Avitia, O. Sepúlveda-Robles, V. Cruz-Sánchez, L. Kameyama, G. Guarneros, A.E. Escalante, Population structure of Pseudomonas aeruginosa through a MLST approach and antibiotic resistance profiling of a Mexican clinical collection, Infect. Genet. Evol. 65 (2018) 43-54.

[14] E. Mahenthiralingam, M.E. Campbell, J. Foster, J.S. Lam, D.P. Speert, Random amplified polymorphic DNA typing of Pseudomonas aeruginosa isolates recovered from patients with cystic fibrosis, J. Clin. Microbiol. 34 (1996) 1129-1135.

[15] V. Waters, J.E. a Zlosnik, Y.C.W. Yau, D.P. Speert, S.D. Aaron, D.S. Guttman, Comparison of three typing methods for Pseudomonas aeruginosa isolates from patients with cystic fibrosis, Eur. J. Clin. Microbiol. Infect. Dis. 31 (2012) 3341-3350.

[16] M.V. Grosso-Becerra, C. Santos-Medellín, A. González-Valdez, J.L. Méndez, G. Delgado, R. Morales-Espinosa, L. Servín-González, L.D. Alcaraz, G. SoberónChávez, Pseudomonas aeruginosa clinical and environmental isolates constitute a single population with high phenotypic diversity, BMC Genomics 15 (2014) 318.

[17] X. Didelot, R. Bowden, D.J. Wilson, T.E. Peto, D.W. Crook, Transforming clinical microbiology with bacterial genome sequencing, Nat. Rev. Genet. 13 (2012) 601-612.

[18] T. Köhler, L.K. Curty, F. Barja, C. van Delden, J.C. Pechère, Swarming of Pseudomonas aeruginosa is dependent on cell-to-cell signaling and requires flagella and pili, J. Bacteriol. 182 (2000) 5990-5996.

[19] N. Verstraeten, K. Braeken, B. Debkumari, M. Fauvart, J. Fransaer, J. Vermant, J. Michiels, Living on a surface: swarming and biofilm formation, Trends Microbiol. 16 (2008) 496-506.

[20] H. Ran, D.J. Hassett, G.W. Lau, Human targets of Pseudomonas aeruginosa pyocyanin, Proc. Natl. Acad. Sci. U.S.A. 100 (2003) 14315-14320.

[21] J.R. Grant, A.S. Arantes, P. Stothard, Comparing thousands of circular genomes using the CGView Comparison Tool, BMC Genomics 13 (2012) 202.

[22] L. Freschi, J. Jeukens, I. Kukavica-Ibrulj, B. Boyle, M.-J. Dupont, J. Laroche, S. Larose, H. Maaroufi, J.L. Fothergill, M. Moore, G.L. Winsor, S.D. Aaron, J. Barbeau, S.C. Bell, J.L. Burns, M. Camara, A. Cantin, S.J. Charette, K. Dewar, É. Déziel, K. Grimwood, R.E.W. Hancock, J.J. Harrison, S. Heeb, L. Jelsbak, B. Jia, D.T. Kenna, T.J. Kidd, J. Klockgether, J.S. Lam, I.L. Lamont, S. Lewenza, N. Loman, F. Malouin, J. Manos, A.G. McArthur, J. McKeown, J. Milot, H. Naghra, D. Nguyen, S.K. Pereira, G.G. Perron, J.-P. Pirnay, P.B. Rainey, S. Rousseau, P.M. Santos, A. Stephenson, V. Taylor, J.F. Turton, N. Waglechner, P. Williams, S.W. Thrane, G.D. Wright, F.S.L. Brinkman, N.P. Tucker, B. Tümmler, C. Winstanley, R.C. Levesque, Clinical utilization of genomics data produced by the international Pseudomonas aeruginosa consortium, Front. Microbiol. 6 (2015) 1036.

[23] J. Klockgether, N. Cramer, L. Wiehlmann, C.F. Davenport, B. Tümmler, Pseudomonas aeruginosa genomic structure and diversity, Front. Microbiol. 2 (2011) 150.

[24] A.N. Hamood, J.A. Colmer, U.A. Ochsner, M.L. Vasil, Isolation and characterization of a Pseudomonas aeruginosa gene, $p t x R$, which positively regulates exotoxin A production, Mol. Microbiol. 21 (1996) 97-110.

[25] V. Dekimpe, E. Déziel, Revisiting the quorum-sensing hierarchy in Pseudomonas aeruginosa: the transcriptional regulator RhlR regulates LasR-specific factors, Microbiology 155 (2009) 712-723.

[26] Y.H. Dong, X.F. Zhang, J.L. Xu, A.T. Tan, L.H. Zhang, VqsM, a novel AraC-type global regulator of quorum-sensing signalling and virulence in Pseudomonas aeruginosa, Mol. Microbiol. 58 (2005) 552-564.

[27] H. Liang, X. Deng, X. Li, Y. Ye, M. Wu, Molecular mechanisms of master regulator VqsM mediating quorum-sensing and antibiotic resistance in Pseudomonas aeruginosa, Nucleic Acids Res. 42 (2014) 10307-10320.

[28] X. Lee, C. Reimmann, G. Greub, J. Sufrin, A. Croxatto, The Pseudomonas aeruginosa toxin L-2-amino-4-methoxy-trans-3-butenoic acid inhibits growth and induces encystment in Acanthamoeba castellanii, Microb. Infect. 14 (2012) 268-272.

[29] T.L. Yahr, A.J. Vallis, M.K. Hancock, J.T. Barbieri, D.W. Frank, ExoY, an adenylate cyclase secreted by the Pseudomonas aeruginosa type III system, Proc. Natl. Acad. Sci. U.S.A. 95 (1998) 13899-13904.

[30] H. Sato, D.W. Frank, ExoU is a potent intracellular phospholipase, Mol. Microbiol. 53 (2004) 1279-1290.

[31] M.H. Diaz, A.R. Hauser, Pseudomonas aeruginosa cytotoxin ExoU is injected into phagocytic cells during acute pneumonia, Infect. Immun. 78 (2010) 1447-1456.

[32] B.I. Kazmierczak, J. Engel, Pseudomonas aeruginosa ExoT acts in vivo as a GTPaseactivating protein for RhoA, Rac1, and Cdc42, Infect. Immun. 70 (2002) 2198-2205.

[33] C.L. Rocha, J. Coburn, E.A. Rucks, J.C. Olson, Characterization of Pseudomonas aeruginosa exoenzyme $S$ as a bifunctional enzyme in J774A.1 macrophages, Infect. Immun. 71 (2003) 5296-5305.

[34] T.R. Walsh, M.A. Toleman, L. Poirel, P. Nordmann, Metallo- $\beta$-lactamases: the quiet before the storm? Clin. Microbiol. Rev. 18 (2005) 306-325.

[35] H.W. Shin, J. Lim, S. Kim, J. Kim, G.C. Kwon, S.H. Koo, Characterization of trimethoprim-sulfamethoxazole resistance genes and their relatedness to class 1 integron and insertion sequence common region in gram-negative bacilli, $\mathrm{J}$. Microbiol. Biotechnol. 25 (2014) 137-142.

[36] P. Cholley, R. Ka, C. Guyeux, M. Thouverez, N. Guessennd, B. Ghebremedhin, T. Frank, X. Bertrand, D. Hocquet, Population structure of clinical Pseudomonas aeruginosa from west and central african countries, PLoS One 9 (2014) e107008.

[37] T.J. Kidd, K.A. Ramsay, S. Vidmar, J.B. Carlin, S.C. Bell, C.E. Wainwright, K. Grimwood, P.W. Francis, C. Dakin, J. Cheney, N. George, C.F. Robertson, M. Moodie, R. Carzino, R. Carter, D.S. Armstrong, P.J. Cooper, K. McKay, A.J. Martin, B. Whitehead, J. Hunter, C.A. Byrnes, H.A. Tiddens, K. Graniel, K. Gerbrands, L. Mott, Pseudomonas aeruginosa genotypes acquired by children with cystic fibrosis by age 5-years, J. Cyst. Fibros. 14 (2015) 361-369.

[38] J. Elias, C. Schoen, G. Heinze, G. Valenza, E. Gerharz, H. Riedmiller, U. Vogel, Nosocomial outbreak of VIM-2 metallo- $\beta$-lactamase-producing Pseudomonas aeruginosa associated with retrograde urography, Clin. Microbiol. Infect. 16 (2010) 1494-1500.

[39] P. Cholley, M. Thouverez, D. Hocquet, N. van der Mee-Marquet, D. Talon, $\mathrm{X}$. Bertrand, Most multidrug-resistant Pseudomonas aeruginosa isolates from hospitals in eastern France belong to a few clonal types, J. Clin. Microbiol. 49 (2011) 2578-2583.

[40] A. Oliver, X. Mulet, C. López-Causapé, C. Juan, The increasing threat of Pseudomonas aeruginosa high-risk clones, Drug Resist. Updates 21 (2015) 41-59.

[41] D.G. Lee, J.M. Urbach, G. Wu, N.T. Liberati, R.L. Feinbaum, S. Miyata, L.T. Diggins, J. He, M. Saucier, E. Déziel, L. Friedman, L. Li, G. Grills, K. Montgomery, R. Kucherlapati, L.G. Rahme, F.M. Ausubel, Genomic analysis reveals that Pseudomonas aeruginosa virulence is combinatorial, Genome Biol. 7 (2006) R90.

[42] R.N. Hvorup, B. Winnen, A.B. Chang, Y. Jiang, X.F. Zhou, M.H. Saier, The multidrug/oligosaccharidyl-lipid/polysaccharide (MOP) exporter superfamily, Eur. J. Biochem. 270 (2003) 799-813.

[43] R.L. Dy, R. Przybilski, K. Semeijn, G.P.C. Salmond, P.C. Fineran, A widespread bacteriophage abortive infection system functions through a Type IV toxin-antitoxin mechanism, Nucleic Acids Res. 42 (2014) 4590-4605.

[44] B. Buchfink, C. Xie, D.H. Huson, Fast and sensitive protein alignment using DIAMOND, Nat. Methods 12 (2015) 59-60.

[45] B. Hu, Y. Nie, S. Geng, X.-L. Wu, Complete genome sequence of the petroleumemulsifying bacterium Pseudomonas stutzeri SLG510A3-8, J. Biotechnol. 211 (2015) 1-2.

[46] K. Baruch, L. Gur-Arie, C. Nadler, S. Koby, G. Yerushalmi, Y. Ben-Neriah, O. Yogev, E. Shaulian, C. Guttman, R. Zarivach, I. Rosenshine, V. Auerbuch, D. Golenbock, R. Isberg, M. Barkett, T. Gilmore, F. Chen, D. Huang, Y. Chen, G. Ghosh, Y. Chen, S. Ghosh, G. Ghosh, Z. Chen, K. Datsenko, B. Wanner, R. Davis, P. Dean, B. Kenny, B. Derijard, M. Hibi, I. Wu, T. Barrett, B. Su, T. Deng, M. Karin, R. Davis, S. Doyle, L. O'Neill, F. Dziva, P. van Diemen, M. Stevens, A. Smith, T. Wallis, R. Eferl, E. Wagner, X. Gao, F. Wan, K. Mateo, E. Callegari, D. Wang, W. Deng, J. Puente, F. Li, M. Chaussee, B. Finlay, M. Lenardo, P. Hardwidge, C. Hemrajani, C. Berger, K. Robinson, O. Marches, A. Mousnier, G. Frankel, M. Hibi, A. Lin, T. Smeal, A. Minden, M. Karin, A. Iguchi, N. Thomson, Y. Ogura, D. Saunders, T. Ooka, I. Henderson, D. Harris, M. Asadulghani, K. Kurokawa, P. Dean, B. Kenny, M. Quail, S. Thurston, G. Dougan, T. Hayashi, J. Parkhill, G. Frankel, D. Jandhyala, T. Rogers, A. Kane, A. Paton, J. Paton, C. Thorpe, H. Jijon, W. Panenka, K. Madsen, H. Parsons, T. Kallunki, B. Su, I. Tsigelny, H. Sluss, B. Derijard, G. Moore, R. Davis, M. Karin, S. Kang, S. Woo, J. Im, J. Yang, C. Yun, H. Ju, C. Son, E. Moon, S. Han, M. Karin, Y. Ben-Neriah, T. Kawai, S. Akira, J. Kucharczak, M. Simmons, Y. Fan, C. Gelinas, O. Marches, S. Wiles, F. Dziva, R. La Ragione, S. Schuller, A. Best, A. Phillips, E. Hartland, M. Woodward, M. Stevens, G. Frankel, E. Miao, D. Mao, N. Yudkovsky, R. Bonneau, C. Lorang, S. Warren, I. Leaf, A. Aderem, C. Nadler, K. Baruch, S. Kobi, E. Mills, G. Haviv, M. Farago, I. Alkalay, S. Bartfeld, T. Meyer, Y. Ben-Neriah, I. Rosenshine, H. Newton, J. Pearson, L. Badea, M. Kelly, M. Lucas, G. Holloway, K. Wagstaff, M. Dunstone, J. Sloan, J. Whisstock, J. Kaper, R. Robins-Browne, D. Jans, G. Frankel, A. Phillips, B. Coulson, E. Hartland, J. Renzing, D. Lane, T. Roger, T. Out, N. Mukaida, K. Matsushima, H. Jansen, R. Lutter, M. RuchaudSparagano, M. Maresca, B. Kenny, S. Shames, W. Deng, J. Guttman, C. de Hoog, Y. Li, P. Hardwidge, H. Sham, B. Vallance, L. Foster, B. Finlay, F. Shao, C. Golstein, J. Ade, M. Stoutemyer, J. Dixon, R. Innes, E. Shaulian, M. Karin, O. Takeuchi, S. Akira, M. Toledano, D. Ghosh, F. Trinh, W. Leonard, C. Tournier, P. Hess, D. Yang, J. Xu, T. Turner, A. Nimnual, D. Bar-Sagi, S. Jones, R. Flavell, R. Davis, A. Yaron, A. Hatzubai, M. Davis, I. Lavon, S. Amit, A. Manning, J. Andersen, M. Mann, F. Mercurio, Y. Ben-Neriah, O. Yogev, S. Anzi, K. Inoue, E. Shaulian, 
O. Yogev, K. Saadon, S. Anzi, K. Inoue, E. Shaulian, Y. Yu, H. Zeng, S. Lyons, A. Carlson, D. Merlin, A. Neish, A. Gewirtz, Metalloprotease type III effectors that specifically cleave JNK and NF-kB, EMBO J. 30 (2011) 221-231.

[47] E. Mowat, S. Paterson, J.L. Fothergill, E.A. Wright, M.J. Ledson, M.J. Walshaw, M.A. Brockhurst, C. Winstanley, Pseudomonas aeruginosa population diversity and turnover in cystic fibrosis chronic infections, Am. J. Respir. Crit. Care Med. 183 (2011) 1674-1679.

[48] F. Duong, E. Bonnet, V. Géli, A. Lazdunski, M. Murgier, A. Filloux, The AprX protein of Pseudomonas aeruginosa: a new substrate for the Apr type I secretion system, Gene 262 (2001) 147-153.

[49] A.J. Laarman, B.W. Bardoel, M. Ruyken, J. Fernie, F.J. Milder, J.A.G. van Strijp, S.H.M. Rooijakkers, Pseudomonas aeruginosa alkaline protease blocks complement activation via the classical and lectin pathways, J. Immunol. 188 (2012) 386-393.

[50] J.C.A. Luckett, O. Darch, C. Watters, M. AbuOun, V. Wright, E. Paredes-Osses, J. Ward, H. Goto, S. Heeb, S. Pommier, K.P. Rumbaugh, M. Cámara, K.R. Hardie, A novel virulence strategy for Pseudomonas aeruginosa mediated by an autotransporter with arginine-specific aminopeptidase activity, PLoS Pathog. 8 (2012) e1002854.

[51] S. Bruchmann, A. Dötsch, B. Nouri, I.F. Chaberny, S. Häußler, Quantitative contributions of target alteration and decreased drug accumulation to Pseudomonas aeruginosa fluoroquinolone resistance, Antimicrob. Agents Chemother. 57 (2013) 1361-1368.

[52] S.C. Woolwine, A.B. Sprinkle, D.J. Wozniak, Loss of Pseudomonas aeruginosa PhpA aminopeptidase activity results in increased $\operatorname{algD}$ transcription, J. Bacteriol. 183 (2001) 4674-4679.

[53] H.-C. Chiu, T.-L. Lin, J.-T. Wang, Identification and characterization of an organic solvent tolerance gene in Helicobacter pylori, Helicobacter 12 (2007) 74-81.

[54] T. Hay, S. Fraud, C.H.-F. Lau, C. Gilmour, K. Poole, Antibiotic inducibility of the MexXY multidrug efflux operon of Pseudomonas aeruginosa: involvement of the MexZ anti-repressor ArmZ, PLoS One 8 (2013) e56858.

[55] J.M. Struble, R.T. Gill, Reverse engineering antibiotic sensitivity in a multidrugresistant Pseudomonas aeruginosa isolate, Antimicrob. Agents Chemother. 50 (2006) 2506-2515.

[56] M.M. Ochs, M.P. McCusker, M. Bains, R.E.W. Hancock, Negative regulation of the Pseudomonas aeruginosa outer membrane porin OprD selective for imipenem and basic amino acids, Antimicrob. Agents Chemother. 43 (1999) 1085-1090.

[57] R.A. Bever, B.H. Iglewski, Molecular characterization and nucleotide sequence of the Pseudomonas aeruginosa elastase structural gene, J. Bacteriol. 170 (1988) 4309-4314.

[58] S.S. Twining, S.E. Kirschner, L.A. Mahnke, D.W. Frank, Effect of Pseudomonas aeruginosa elastase, alkaline protease, and exotoxin A on corneal proteinases and proteins, Investig. Ophthalmol. Vis. Sci. 34 (1993) 2699-2712.

[59] D.J. Wolter, P.D. Lister, Mechanisms of $\beta$-lactam resistance among Pseudomonas aeruginosa, Curr. Pharmaceut. Des. 19 (2013) 209-222.

[60] J.L. Malloy, Pseudomonas aeruginosa protease IV degrades surfactant proteins and inhibits surfactant host defense and biophysical functions, AJP Lung Cell, Mol. Physiol. 288 (2004) L409-L418.

[61] F. Casilag, A. Lorenz, J. Krueger, F. Klawonn, S. Weiss, S. Haussler, The LasB elastase of Pseudomonas aeruginosa acts in concert with alkaline protease AprA to prevent flagellin-mediated immune recognition, Infect. Immun. 84 (2015) 162-171.

[62] R. Cahan, I. Axelrad, M. Safrin, D.E. Ohman, E. Kessler, A secreted aminopeptidase of Pseudomonas aeruginosa: identification, primary structure, and relationship to other aminopeptidases, J. Biol. Chem. 276 (2001) 43645-43652.

[63] N. Kanayama, Y. Kajiwara, J. Goto, E. el Maradny, K. Maehara, K. Andou, T. Terao, Inactivation of interleukin-8 by aminopeptidase N (CD13), J. Leukoc. Biol. 57 (1995) 129-134.

[64] B.W. Bardoel, D. Hartsink, M.M. Vughs, C.J.C. de Haas, J.A.G. van Strijp, K.P.M. van Kessel, Identification of an immunomodulating metalloprotease of Pseudomonas aeruginosa (IMPa), Cell, An. Microbiol. 14 (2012) 902-913.
[65] I. Gentschev, G. Dietrich, W. Goebel, The E. coli $\alpha$-hemolysin secretion system and its use in vaccine development, Trends Microbiol. 10 (2002) 39-45.

[66] A.L. Pimenta, K. Racher, L. Jamieson, M.A. Blight, I.B. Holland, Mutations in HlyD, part of the type 1 translocator for hemolysin secretion, affect the folding of the secreted toxin, J. Bacteriol. 187 (2005) 7471-7480.

[67] H. Akama, T. Matsuura, S. Kashiwagi, H. Yoneyama, S.I. Narita, T. Tsukihara, A. Nakagawa, T. Nakae, Crystal structure of the membrane fusion protein, MexA, of the multidrug transporter in Pseudomonas aeruginosa, J. Biol. Chem. 279 (2004) 25939-25942.

[68] L. Fito-Boncompte, A. Chapalain, E. Bouffartigues, H. Chaker, O. Lesouhaitier, G. Gicquel, A. Bazire, A. Madi, N. Connil, W. Véron, L. Taupin, B. Toussaint, P. Cornelis, Q. Wei, K. Shioya, E. Déziel, M.G.J. Feuilloley, N. Orange, A. Dufour, S. Chevalier, Full virulence of Pseudomonas aeruginosa requires OprF, Infect. Immun. 79 (2011) 1176-1186.

[69] E. Bouffartigues, J.A. Moscoso, R. Duchesne, T. Rosay, L. Fito-Boncompte, G. Gicquel, O. Maillot, M. Bénard, A. Bazire, G. Brenner-Weiss, O. Lesouhaitier, P. Lerouge, A. Dufour, N. Orange, M.G.J. Feuilloley, J. Overhage, A. Filloux, S. Chevalier, The absence of the Pseudomonas aeruginosa OprF protein leads to increased biofilm formation through variation in c-di-GMP level, Front. Microbiol. 6 (2015) 630.

[70] A.B. Taylor, B.S. Smith, S. Kitada, K. Kojima, H. Miyaura, Z. Otwinowski, A. Ito, J. Deisenhofer, Crystal structures of mitochondrial processing peptidase reveal the mode for specific cleavage of import signal sequences, Structure 9 (2001) 615-625.

[71] J.V. King, W.G. Liang, K.P. Scherpelz, A.B. Schilling, S.C. Meredith, W.J. Tang, Molecular basis of substrate recognition and degradation by human presequence protease, Structure 22 (2014) 996-1007.

[72] J.P. Pearson, M. Feldman, B.H. Iglewski, A. Prince, Pseudomonas aeruginosa cell-tocell signaling is required for virulence in a model of acute pulmonary infection, Infect. Immun. 68 (2000) 4331-4334.

[73] K. Winzer, P. Williams, Quorum sensing and the regulation of virulence gene expression in pathogenic bacteria, Int. J. Med. Microbiol. 291 (2001) 131-143.

[74] M. Toyofuku, N. Nomura, T. Fujii, N. Takaya, H. Maseda, I. Sawada, T. Nakajima, H. Uchiyama, Quorum sensing regulates denitrification in Pseudomonas aeruginosa PAO1, J. Bacteriol. 189 (2007) 4969-4972.

[75] M. Schobert, D. Jahn, Anaerobic physiology of Pseudomonas aeruginosa in the cystic fibrosis lung, Int. J. Med. Microbiol. 300 (2010) 549-556.

[76] S. Chugani, B.S. Kim, S. Phattarasukol, M.J. Brittnacher, S.H. Choi, C.S. Harwood, E.P. Greenberg, Strain-dependent diversity in the Pseudomonas aeruginosa quorumsensing regulon, Proc. Natl. Acad. Sci. U.S.A. 109 (2012) E2823-E2831.

[77] H. Zhu, R. Bandara, T.C.R. Conibear, S.J. Thuruthyil, S.A. Rice, S. Kjelleberg, M. Givskov, M.D.P. Willcox, Pseudomonas aeruginosa with LasI quorum-sensing deficiency during corneal infection, Investig. Ophthalmol. Vis. Sci. 45 (2004) 1897-1903.

[78] P. Vidya, L. Smith, T. Beaudoin, Y.C.W. Yau, S. Clark, B. Coburn, D.S. Guttman, D.M. Hwang, V. Waters, Chronic infection phenotypes of Pseudomonas aeruginosa are associated with failure of eradication in children with cystic fibrosis, Eur. J. Clin. Microbiol. Infect. Dis. 35 (2016) 67-74.

[79] C.-R. Huang, S.-F. Chen, C.-H. Lu, Y.-C. Chuang, N.-W. Tsai, C.-C. Chang, H.C. Wang, C.-C. Chien, W.-N. Chang, Clinical characteristics and therapeutic outcomes of nosocomial super-infection in adult bacterial meningitis, BMC Infect. Dis. 11 (2011) 133.

[80] C. Cillóniz, S. Ewig, R. Menéndez, M. Ferrer, E. Polverino, S. Reyes, A. Gabarrús, M.A. Marcos, J. Cordoba, J. Mensa, A. Torres, Bacterial co-infection with H1N1 infection in patients admitted with community acquired pneumonia, J. Infect. 65 (2012) 223-230.

[81] D.A. Soborg, N.B. Hendriksen, M. Kilian, N. Kroer, Widespread occurrence of bacterial human virulence determinants in soil and freshwater environments, Appl. Environ. Microbiol. 79 (2013) 5488-5497. 